\title{
The zenithal 4-m International Liquid Mirror Telescope: a unique facility for supernova studies
}

\author{
Brajesh Kumar, ${ }^{1 \star}$ Kanhaiya L. Pandey, ${ }^{1} \dagger$ S. B. Pandey,${ }^{2} \ddagger$ P. Hickson, ${ }^{3}$ E. F. Borra, ${ }^{4}$ \\ G. C. Anupama ${ }^{1}$ and J. Surdej ${ }^{5}$ \\ ${ }^{1}$ Indian Institute of Astrophysics, II Block, Koramangala, Bangalore 560 034, India \\ ${ }^{2}$ Aryabhatta Research Institute of Observational Sciences, Manora Peak, Nainital 263 002, India \\ ${ }^{3}$ Department of Physics and Astronomy, University of British Columbia, 6224 Agricultural Road, Vancouver, BC V6T 1Z1, Canada \\ ${ }^{4}$ Department of Physics, Université Laval, 2325, rue de l'Université, Québec, G1V 0A6, Canada \\ ${ }^{5}$ Institut d'Astrophysique et de Géophysique, Université de Liège, Allée du 6 Août 19, Bât B5C, 4000 Liège, Belgium \\ Accepted $\longrightarrow$, Received $\longrightarrow$; in original form $\longrightarrow$
}

\begin{abstract}
The 4-m International Liquid Mirror Telescope (ILMT) will soon become operational at the newly developed Devasthal observatory near Nainital (Uttarakhand, India). Coupled with a $4 \mathrm{k} \times 4 \mathrm{k}$ pixels CCD detector and TDI optical corrector, it will reach approximately 22.8 , 22.3 and 21.4 magnitude in the $g^{\prime}, r^{\prime}$ and $i^{\prime}$ spectral bands, respectively in a single scan. The limiting magnitudes can be further improved by co-adding the consecutive night images in particular filters. The uniqueness to observe the same sky region by looking towards the zenith direction every night, makes the ILMT a unique instrument to detect new supernovae ( $\mathrm{SNe})$ by applying the image subtraction technique. High cadence $(\sim 24$ hours $)$ observations will help to construct dense sampling multi-band SNe light curves. We discuss the importance of the ILMT facility in the context of SNe studies. Considering the various plausible cosmological parameters and observational constraints, we perform detailed calculations of the expected $\mathrm{SNe}$ rate that can be detected with the ILMT in different spectral bands.
\end{abstract}

Key words: Supernovae: general - instrumentation: miscellaneous - techniques: image processing - telescopes

\section{INTRODUCTION}

The new generation large area survey programs have contributed with their invaluable data to discover new supernovae ( $\mathrm{SNe}$ ). In order to detect such transients, two different strategies are typically applied. First is the pointed survey approach where a fixed catalogue of galaxies are independently observed with a cadence of a few days. In the second method, a specific area of the sky is surveyed very frequently and transients are identified using the image subtraction technique. Some important $\mathrm{SNe}$ search programs based on these methods are: the Lick Observatory Supernova Search (LOSS; Li et al. 2000), the Panoramic Survey Telescope \& Rapid Response System (Pan-STARRS; Kaiser et al. 2002), the Dark Energy Survey (DES; Abbott et al. 2005), the Canada-France-Hawaii Telescope-Legacy Survey (CFHT-LS; Astier et al. 2006), the Carnegie Supernova Project (CSP; Hamuy et al. 2006) the Equation of State: SupErNovae trace Cosmic Expansion (ESSENCE;

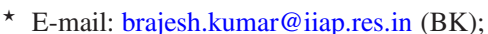

$\dagger$ kanhaiya.pandey@iiap.res.in (KLP);

† shashi@aries.res.in (SBP)
}

Miknaitis et al. 2007; Wood-Vasey et al. 2007), the Sloan Digital Sky Survey (SDSS; Frieman et al. 2008; Sako et al. 2008), the Catalina Real-Time Transient Survey (CRTS; Drake et al. 2009), the Palomar Transient Factory (PTF; Law et al. 2009), the AllSky Automated Survey for SuperNovae (ASAS-SN; Shappee et al. 2014) and the upcoming Zwicky Transient Facility (ZTF; Bellm 2014).

It is notable that despite their great contribution to supernova (SN) search, this kind of projects are observationally expensive, requiring many hours of valuable telescope time to complete, and are also depth and cadence limited. Furthermore, the majority of them do not perform observations of the same strip of sky on a regular basis every night (however, see ASAS-SN survey, http://www . astronomy . ohio-state. edu/asassn/index. shtml). The unconventional Liquid Mirror Telescopes (LMTs, Borra et al. 1985, 1992b; Hickson et al. 1993) may provide a unique way to overcome some of these issues in a certain fashion. For SNe studies, LMT observations are useful over the generic facilities in several aspects:

- Unbiased imaging: Most nearby SNe are discovered by re- 
peated imaging of catalogued galaxies (Filippenko et al. 2001) which introduces a possible bias towards metal rich galaxies. Though ongoing ASAS-SN survey like programs have improved the situation and much is expected with the upcoming ZTF facility. Moreover, a LMT will image the same strip of sky passing over it without any selection bias.

- Inexpensive technology: The cost of constructing a moderate aperture telescope (4-m diameter) is roughly $1 / 50$ that of a conventional telescope of the same class (Borra 2001a, 2003).

- Continuous data flow: There will be no loss of precious observing time because a LMT will observe continuously during the nights except for bad weather or technical problems and produce a large amount of scientific data using the sky light.

- Easy image pre-processing: Unlike conventional imaging, here image pre-processing is comparatively easier. For example, the image reduction is performed by dividing each column by a one-dimensional flat field. That can be achieved directly from the scientific data.

- Deeper imaging: Since each night the same sky strip will be captured by the telescope, we can co-add the consecutive night data to produce deeper images.

Different size LMTs have already been built and operated by several groups (for example; Borra et al. 1989; Hickson et al. 1994) and the 6-m diameter Large Zenithal Telescope (Hickson et al. 2007) was the largest one in its class. The scientific contributions of these facilities were mainly limited due to the lack of an appropriate TDI corrector and/or large CCD camera and/or location (c.f. poor sky conditions). Therefore, a full time LMT entirely dedicated to astronomical observations was proposed and the idea of building the International Liquid Mirror Telescope (ILMT ${ }^{1}$ ) was born. SNe related study is one of the major scientific interests behind the ILMT project (Surdej et al. 2006).

A link between the star formation history and the cosmic supernova rate has been an open question. It is generally believed that Type Ia $\mathrm{SNe}$ originate from the intermediate mass $\left(3-8 \mathrm{M}_{\odot}\right)$ and both young and old population stars (Wang \& Han 2012; Maoz, Mannucci \& Nelemans 2014, for recent reviews). Such explosions are supposed to be the consequences of thermonuclear disruptions when a carbon-oxygen white dwarf reaches the critical Chandrasekhar mass limit $\left(\simeq 1.4 \mathrm{M}_{\odot}\right.$, Chandrasekhar 1931) by accreting matter from its evolving binary companion (Hoyle \& Fowler 1960; Whelan \& Iben 1973; Woosley, Taam \& Weaver 1986; Hillebrandt \& Niemeyer 2000). The observational features of these events show homogeneity and due to their high luminosity near the maximum light, they are detectable at high redshift (Filippenko 2005). Type Ia SNe are considered to be reliable standard candles (Branch \& Tammann 1992; Phillips 1993) and play an important role to constrain the geometry of the Universe (e.g. Riess et al. 1998; Perlmutter et al. 1999; Riess et al. 2007).

Contrary to the progenitors of Type Ia $\mathrm{SNe}$, massive stars $\left(M \geqslant 8 \mathrm{M}_{\odot}\right)$ follow a different evolutionary path (e.g. Branch et al. 1991; Heger et al. 2003; Smartt 2009; Langer 2012). At the final stage when their nuclear fuel is exhausted, the gravitational collapse of the stellar core triggers into a catastrophic death that appears in the form of a core-collapse supernova (CCSN). The spectro-photometric features led to classify them in several types (c.f. IIP, IIL, IIn, IIb, Ib, Ic and Ic-BL; see Minkowski 1941;
Filippenko 1997, for a review). CCSNe show diverse observational properties. For example, their absolute magnitude distribution peaks roughly 1.5 mag fainter than $\mathrm{SN}$ Ia and covers a range of more than 5 mag (Richardson et al. 2002, 2006). Similarly, a wide dispersion is seen in the ejecta mass, kinetic energy, radiated energy and the amount of synthesized radioactive materials created in the explosion. This indicates that possibly different physical mechanisms play an important role during the evolution phases of the progenitors such as stellar wind (Puls, Vink \& Najarro 2008), mass transfer in a binary system (Wheeler \& Levreault 1985; Podsiadlowski, Joss \& Hsu 1992; Yoon, Woosley \& Langer 2010; Sana et al., 2012) and mass loss (Smith \& Owocki 2006; Smith 2014), etc.

Along with astrophysical and cosmological implications, $\mathrm{SNe}$ are also primarily responsible for the chemical enrichment of galaxies through their heavy elements and dust (e.g. Matteucci \& Greggio 1986; Todini \& Ferrara 2001; Bianchi \& Schneider 2007). Furthermore, the expanding shock waves produced during the explosion sweep, compress and heat the surrounding interstellar medium that finally triggers the star formation process (e.g. Herbst \& Assousa 1977; Elmegreen 1998, and references therein). Unbiased and large sample studies of $\mathrm{SNe}$ may provide answers to some of the underlying questions related to the star formation history, progenitor evolution scenario and parameters causing the diversity in their observed properties. In this context the ILMT deep imaging survey along with complementary observations from other existing observational facilities will be advantageous.

\section{THE ILMT PROJECT}

The ILMT project is a scientific collaboration between four countries: Belgium, India, Canada and Poland. The main participating institutions are: the Liège Institute of Astrophysics and Geophysics (University of Liège, Belgium), the Aryabhatta Research Institute of Observational Sciences (ARIES, India), the Royal Observatory of Belgium, several Canadian universities (British Columbia, Laval, Montréal, Toronto, Victoria and York) and the Observatory of Poznan (Poland). The AMOS (Advanced Mechanical and Optical Systems) company in Belgium has participated in the manufacturing of the telescope.

The ILMT is being installed at the Devasthal (meaning 'Abode of God') mountain peak, in the central Himalayan range in India. This is a newly developed observatory under ARIES. A panaromic view of the site is illustrated in Fig. 1. The Devasthal observatory is situated at an altitude of $\sim 2450 \mathrm{~m}$, with longitude $79^{\circ} 41^{\prime} 04^{\prime \prime}$ East and latitude $+29^{\circ} 21^{\prime} 40^{\prime \prime}$ (c.f. Sagar et al. 2011, 2012). It is important to highlight that in view of the site advantages, apart from the upcoming 4-m ILMT, the other existing astronomical observing facilities at Devasthal are the 1.3-m $D F O T^{2}$ and 3.6- $\mathrm{m} \mathrm{DOT}^{3}$. Major scientific prospectives of these telescopes can be found in Sagar (2016, and references therein).

A sketch of the ILMT structure is shown in Fig. 1 (right panel). It consists of three major parts, namely the air bearing, the container and the vertical structure which will hold the corrector and $\mathrm{CCD}$ camera. The primary mirror is a 4-m diameter epoxy-carbonfiber structure that has a smooth parabolic upper surface produced 

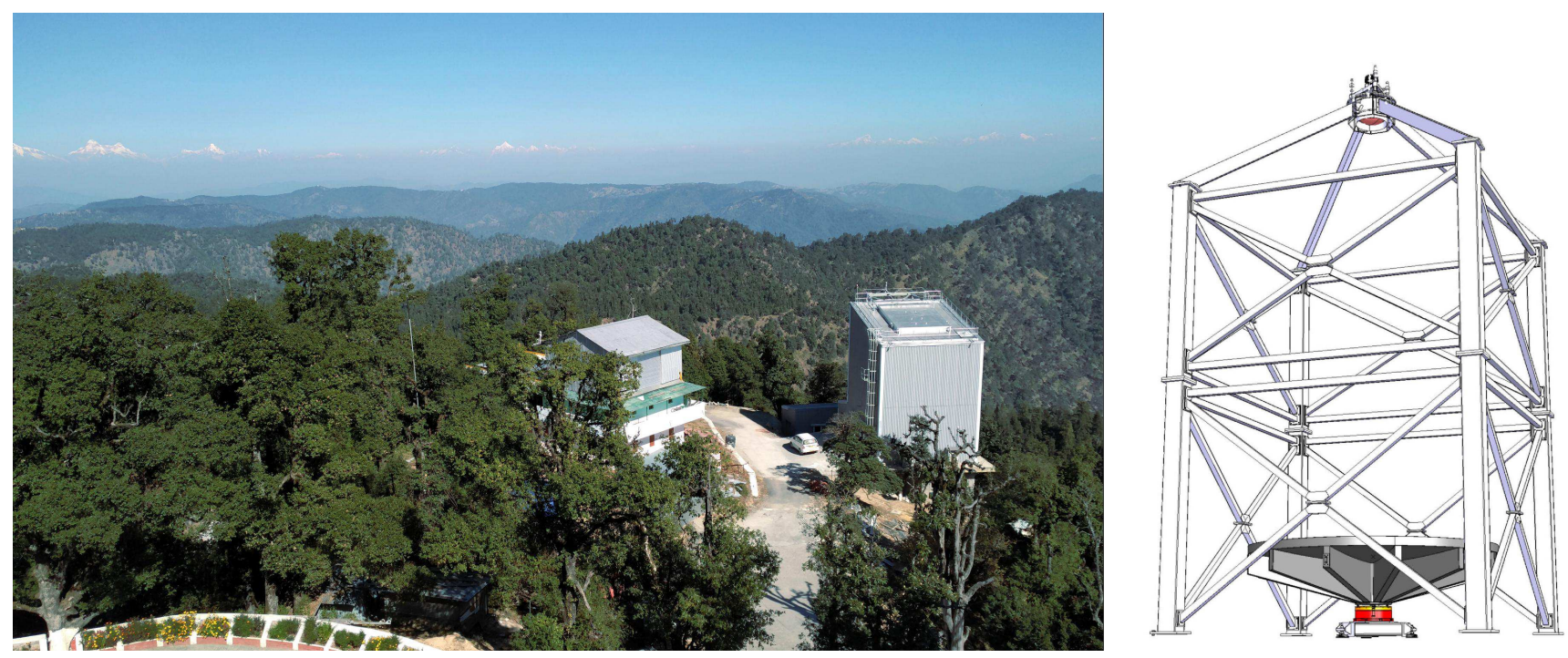

Figure 1. Left panel: Panoramic view of the ILMT site. The 1.3-m DFOT and the 4-m ILMT are in the middle and right side, respectively in this image. Right panel: Major components of the ILMT. Here, the container is gray, the air bearing is red, the three-point mount (white) sits below the air bearing and the vertical steel frames (white) hold the corrector and the CCD camera at the top. The tentative size and other parameters of the telescope are listed in Table 1. Note the nice view on the Himalayan chain in the background of the left photograph.

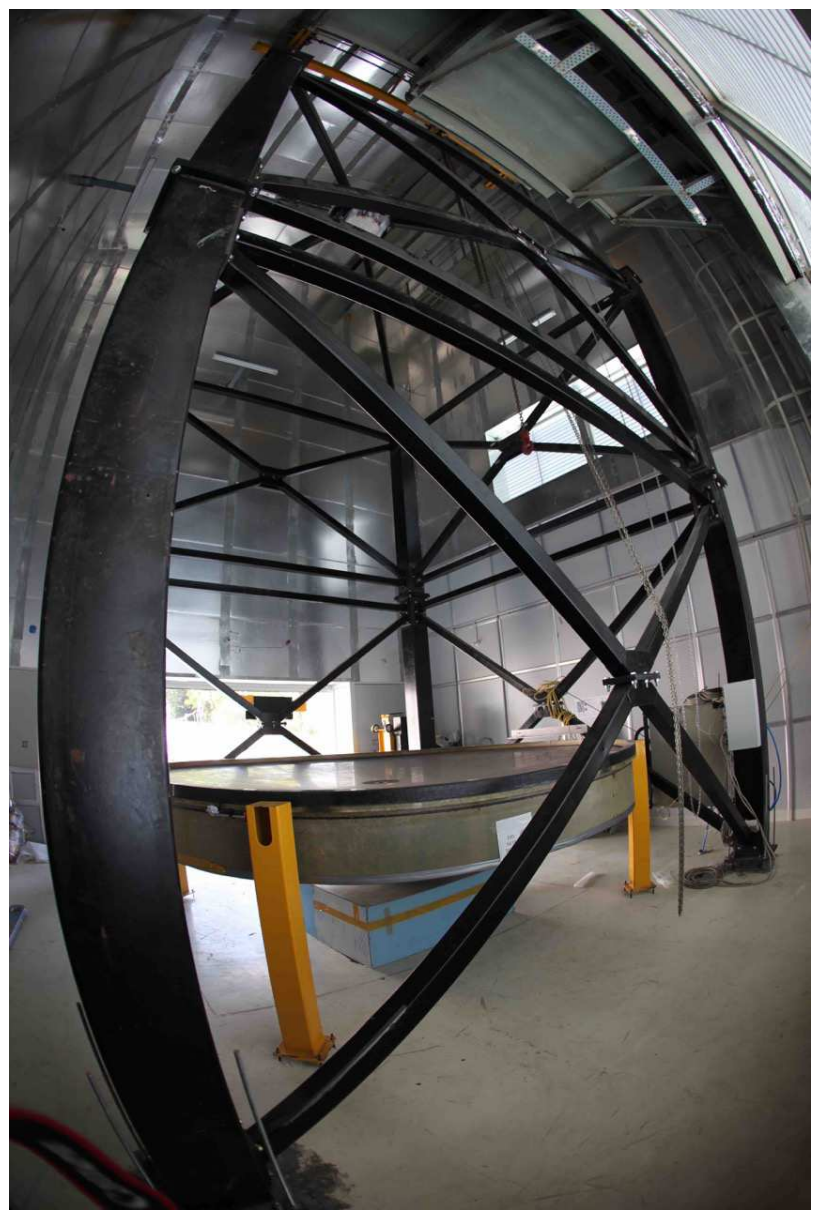

Figure 2. Fish eye view of the present status of the ILMT. To protect from the dust, the air bearing and the three-point mount are covered with a wooden box (blue colour). Four safety pillars (yellow colour) are also visible near the parabolic container to prevent any mercury spill. by spin casting (Magette 2010; Kumar 2014), see Fig. 2. The dish will support a thin layer (approximately $2-3 \mathrm{~mm}$ thick) of liquid mercury that will produce the reflecting surface. When the mirror is rotated uniformly about its vertical axis, the combination of gravity and centrifugal force will produce an equilibrium surface that is parabolic to high accuracy. A detailed explanation can be found in Borra (1982). Although mercury vapour is harmful, it is greatly suppressed by a thin transparent layer of oxide that forms soon after emplacement. Moreover, a thin film of mylar ${ }^{4}$, co-rotating with the mirror, will contain any remaining vapour. This film is required to prevent vortices, produced in the boundary layer above the mirror due to its rotation, from disturbing the liquid surface.

The ILMT is a zenithal rotating telescope. It cannot track stellar objects like conventional glass mirror telescopes. Therefore, images are secured by electronically stepping the relevant CCD charges. The transfer rate is kept similar as the target drifts across the detector (i.e. equal to the observatory sidereal rate). This specific technique is known as the Time Delayed Integration (TDI) or drift-scanning (see Gibson \& Hickson 1992, and references therein). Advantages of the TDI mode of observations can be found in Kumar (2014, and references therein). Because the primary mirror is parabolic, a glass corrector will be used to obtain a good image quality over a field of view of 27 arcmin in diameter including TDI correction (see Hickson \& Richardson 1998; Vangeyte et al. 2002). A CCD detector (4096 $\times 4096$ pixels) manufactured by 'Spectral Instruments' will be positioned at the prime focus, located about $8 \mathrm{~m}$ above the mirror. The ILMT observations will be mainly performed with the $i^{\prime}$ filter (although there are additional filters $g^{\prime}$ and $r^{\prime}$ ). This will be advantageous for a maximum number of nights because the spectral range covered by the $i^{\prime}$ filter is less sensitive to the bright phases of the moon. Initially the ILMT project will be for 5 years which will allow us to collect a large sample of stellar objects including transients. More detailed

${ }^{4}$ It is a scientific grade polyester film (thickness $<12 \mu \mathrm{m}$ ). Optical quality tests of such films for the LMTs are discussed in Borra et al. (1992a); Hickson \& Racine (2007). 
information about its instruments and science cases can be found elsewhere (e.g. Surdej et al. 2006, 2018; Magette 2010; Poels et al. 2012; Finet 2013; Kumar 2014; Kumar et al. 2015, 2018, and references therein).

During the last few years, several experiments have been performed to sort out technical difficulties related to the ILMT. In continuation of such activities we have also performed TDI mode observations from the Devasthal observatory using the $1.3 \mathrm{~m}$ DFOT. These images have been used to test the ILMT data reduction pipeline and preliminary results are presented in Pradhan et al. (2018). The installation process of the telescope began in 2017 March and is now in its final stage. The metallic structure (to hold the CCD camera and corrector), safety pillars, air bearing are already erected. To ensure optimal and very safe operation of the air bearing two parallel air supply systems have been installed. In addition, several components/instruments like pneumatic valves, air dryers, air filters and sensors (pressure, temperature, humidity and dew-point) are also installed. First light of the ILMT is expected before the beginning of the 2018 Monsoon season. For the present status of the ILMT project, see Surdej et al. (2018). A fish eye view of the installation is shown in Fig. 2.

\section{ILMT LIMITING MAGNITUDES AND ACCESSIBLE SKY AREA}

The scientific performance of an instrument depends on the maximization of its throughput. Considering various parameters (e.g. transmission coefficients from the mirrors, filters, CCD glass, sky, extinction and quantum efficiency of the CCD chip), the expected counts $\left(N_{\mathrm{e}}\right)$ from a star of certain magnitude $(\mathrm{m})$ can be estimated using the following formula (McLean 1989; Mayya 1991).

$$
N_{\mathrm{e}}=3.95 \times 10^{11} D^{2} \lambda_{\mathrm{n}} \Delta \lambda_{\mathrm{n}} F_{0}^{\mathrm{n}} 10^{-0.4 \mathrm{~m}} A_{\mathrm{F}} \eta
$$

where $\mathrm{D}$ is the diameter of the telescope, $\lambda_{\mathrm{n}}$ and $\Delta \lambda_{\mathrm{n}}$ are the effective wavelength and bandwidth of the filters, $F_{0}^{\mathrm{n}}$ is the flux density (per wavelength) from a star of magnitude 0 at the wavelength $\lambda_{\mathrm{n}}$ above the Earth atmosphere, $A_{\mathrm{F}}$ is the fractional reflecting area of the mirror surface and $\eta$ is the efficiency of the system (mirror + filter + CCD)

Assuming that each optical photon is capable of producing a corresponding photo-electron, the full well capacity of the required CCD pixel could be estimated by assuming a certain integration time for a star with a known brightness. Furthermore, if the sky brightness is known for a given CCD, we can also calculate the sky counts and the underlying noise.

$$
N=\sqrt{\left(N_{\mathrm{e}} e_{\mathrm{t}}+S_{\mathrm{e}} e_{\mathrm{t}} n_{\mathrm{p}}+D_{\mathrm{c}} e_{\mathrm{t}} n_{\mathrm{p}}+R_{\mathrm{n}}^{2} n_{\mathrm{p}}\right)}
$$

Here, $N_{\mathrm{e}}$ indicates the number of electrons (per sec), $e_{\mathrm{t}}$ the exposure time (sec), $S_{\mathrm{e}}$ the sky brightness (in electrons), $n_{\mathrm{p}}$ the number of pixels in the image of the observed star, $D_{\mathrm{c}}$ the dark current ( $\left.\mathrm{e}^{-} / \mathrm{pix} / \mathrm{sec}\right)$ and $R_{\mathrm{n}}$ the read out noise.

The signal-to-noise ratio (SNR) can also be calculated for stars with different brightness (McLean 1989).

$$
\mathrm{SNR}=\left(\frac{N_{e} \times e_{t}}{N}\right)
$$

The CCD readout noise is Gaussian while the star counts, dark counts are Poisson in nature. The aperture to calculate the star light
Table 1. Different parameters used to calculate the ILMT limiting magnitude. See also Finet (2013).

\begin{tabular}{ll}
\hline Diameter & $4.0-\mathrm{m}$ \\
Fraction of reflecting area & 0.95 \\
Reflectivity & 0.77 \\
Mylar transmission & 0.80 \\
Corrector transmission & 0.85 \\
FWHM & $1.5^{\prime \prime}$ \\
CCD pixel size & $0.4^{\prime \prime} / \mathrm{pixel}$ \\
CCD dark noise & $0.00083 \mathrm{e}^{-} / \mathrm{pixel} / \mathrm{sec}$ \\
CCD readout noise & $5.0 \mathrm{e}^{-}$ \\
CCD gain & $4.0 \mathrm{e}^{-} / \mathrm{ADU}$ \\
Wavelength $\left(g^{\prime}, r^{\prime}, i^{\prime}\right)$ & $4750,6250,7630 \AA$ \\
Wavelength FWHM $\left(g^{\prime}, r^{\prime}, i^{\prime}\right)$ & $1450,1500,1500 \AA$ \\
Extinction $\left(\sim g^{\prime}, \sim r^{\prime}, \sim i^{\prime}\right)$ & $0.21,0.13,0.08 \mathrm{mag}$ \\
Sky mag $\left(\sim g^{\prime}, \sim r^{\prime}, \sim i^{\prime}\right)$ & $21.3,20.5,18.9 \mathrm{mag} / \operatorname{arcsec}^{2}$ \\
CCD quantum efficiency $\left(g^{\prime}, r^{\prime}, i^{\prime}\right)$ & $0.70,0.91,0.91$ \\
Filter transmission $\left(g^{\prime}, r^{\prime}, i^{\prime}\right)$ & $0.92,0.95,0.95$ \\
System efficiency, $\eta\left(g^{\prime}, r^{\prime}, i^{\prime}\right)$ & $0.55,0.74,0.74$ \\
\hline
\end{tabular}

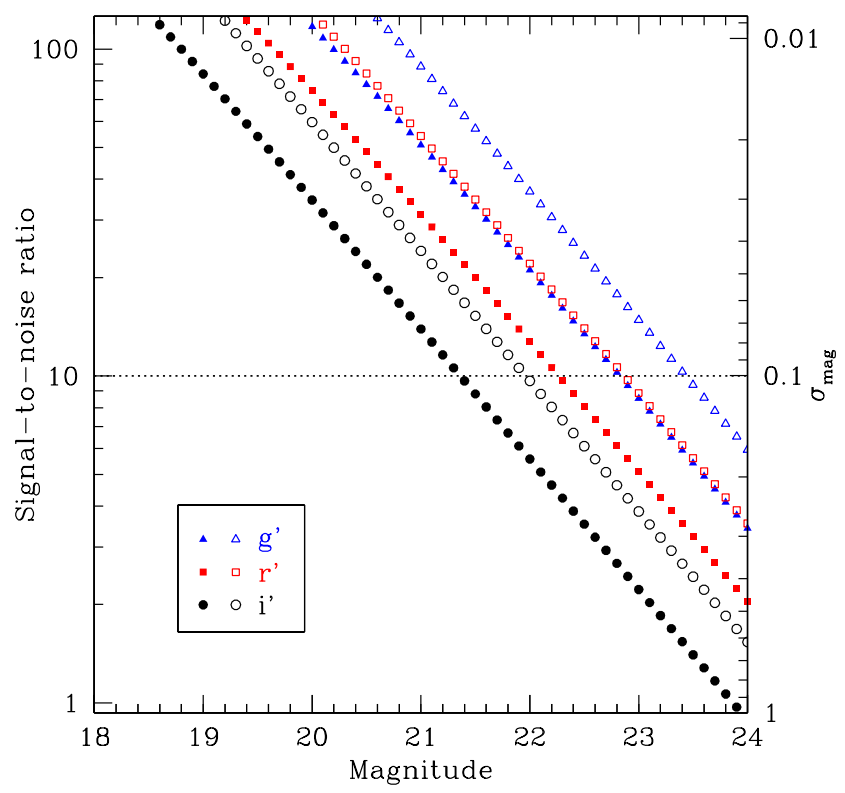

Figure 3. The ILMT limiting magnitudes for the $g^{\prime}, r^{\prime}$ and $i^{\prime}$ filters are shown with different symbols. The $\mathrm{X}$-axis represents the limiting magnitude and the Y-axis represents the SNR and the corresponding error in magnitude. The filled and open symbols represent the results for the exposure of a single scan $(102 \mathrm{sec})$ and three scans (306 sec), respectively (see Section 3 for details). The dotted horizontal line is indicative for a SNR of 10 and an uncertainty of $0.1 \mathrm{mag}$. Approximately $0.5 \mathrm{mag}$ is gained once we stack images taken over three nights in any single filter.

is considered as circular. For the present calculations, the FWHM is considered as 1.5 arcsec, nearly equal to the median seeing at Devasthal. The optimal aperture is considered to be $1 \times$ FWHM (see Howell 1989, 2000). We can also estimate the corresponding error in the magnitude estimation by knowing the value of the SNR (Deep et al. 2011)

$$
\sigma_{\text {mag }}=2.5 \times \log _{10}[1+1 / \mathrm{SNR}] .
$$

We have estimated the limiting magnitudes of the ILMT for 
different filters $\left(g^{\prime}, r^{\prime}\right.$ and $\left.i^{\prime}\right)$ using the above equations. The various parameters used for these estimations are listed in Table 1 . The limiting magnitudes estimated using the above methods for different filters are plotted in Fig. 3 with different symbols. It is obvious from this figure that with an exposure time of $102 \mathrm{sec}$, the limiting magnitudes are $\sim 22.8, \sim 22.3$ and $\sim 21.4$ mag for the $g^{\prime}, r^{\prime}$ and $i^{\prime}$ filters, respectively. Furthermore, since during each night the same strip of sky will pass over the telescope (except for a 4 min. shift in right ascension); successive night images can then be co-added. This will yield a longer effective integration time. Therefore, we have also estimated the limiting magnitudes for $306 \mathrm{sec}$ ( 3 night images) exposure time, using the same parameters. The estimated magnitude limit improves to $\sim 23.4, \sim 22.8$ and $\sim 22.0$ mag for the $g^{\prime}, r^{\prime}$ and $i^{\prime}$ filters, respectively. The co-addition technique is not limited only for 3 nights but it can also be applied for several more night imaging data. Consequently, we may reach very faint magnitude levels (see also Borra 2001a,b, 2003).

Pointing towards the zenith, the ILMT field-of-view (FOV) is centered at the Devasthal observatory latitude which is $29.36^{\circ} \mathrm{N}$. The ILMT FOV is approximately $27^{\prime}$ by $27^{\prime}$. One can find that the total accessible sky area with the ILMT will be 141.2 square degrees. Out of it only $1 / 3$ nightly strip ( $\sim 7$ square degrees) can be monitored. At high galactic latitudes $\left(|\mathrm{b}|>30^{\circ}\right)$ the detection of fainter and more distant objects (e.g. SNe, galaxies, quasars ...) will be possible (see Surdej et al. 2006; Magette 2010; Finet 2013; Kumar 2014).

\section{SUPERNOVA RATE AND ILMT}

The astronomical community is deeply interested in understanding the nature of the different kinds of $\mathrm{SNe}$ and their evolution with redshift. The CCSNe rate is expected to reflect the star-formation rate, increasing with redshift as $(1+z)^{\beta}$ (for $z \approx 0.5$ ) where $\beta$ is in the range 2.5 to 3.9 (see Hopkins 2004; Le Floc'h et al. 2005; Schiminovich et al. 2005; Hopkins \& Beacom 2006; Rujopakarn et al. 2010; Cucciati et al. 2012). The type Ia SNe rate rise is rather slow with redshift, $\sim(1+z)^{\beta}$ (see Pritchet et al. 2008; Perrett et al. 2012, and references therein), where $\beta$ is $2.11 \pm 0.28$ up to $z \sim 1$. In order to search for a possible correlation between the star formation and $\mathrm{SN}$ rates in the local universe, several studies have been performed (see, e.g. Dahlen et al. 2004; Neill et al. 2006; Dilday et al. 2008; Graur et al. 2011; Taylor et al. 2014; Cappellaro et al. 2015; Botticella et al. 2017, and references therein).

In the framework of supernova studies with liquid mirror telescopes, Borra (2001a,b, 2003) has described the cosmological implications of SNe and estimated the number of SNe for a strip of sky using the expected rate of SNe given in Pain et al. (1996). In the following we have performed a detailed calculation of the expected number of SN events which can be detected with the ILMT. We calculate the detection rate for the core-collapse and Type Ia SNe for all the three proposed bands of the ILMT (see Figs. 4 and 5). For the calculations we follow the prescription given in Lien \& Fields (2009). In the following we present a brief description of the steps and the quantities involved in the calculations.

The supernova detection rate per unit redshift per unit solid angle in a filter band $x$ can be expressed as follows,

$$
\frac{d N_{S N, o b s, x}}{d t_{o b s} d z d \Omega}=R_{\mathrm{SN}}(z) f_{\text {detect }}\left(z ; m_{\text {lim }, x}^{\mathrm{SN}}\right) \frac{r(z)^{2}}{1+z} \frac{d r}{d z}
$$

where $r(z)$ is the co-moving distance, $R_{S N}(z)$ is the cosmic $\mathrm{SN}$ rate

MNRAS 000, 1-10 (2018)
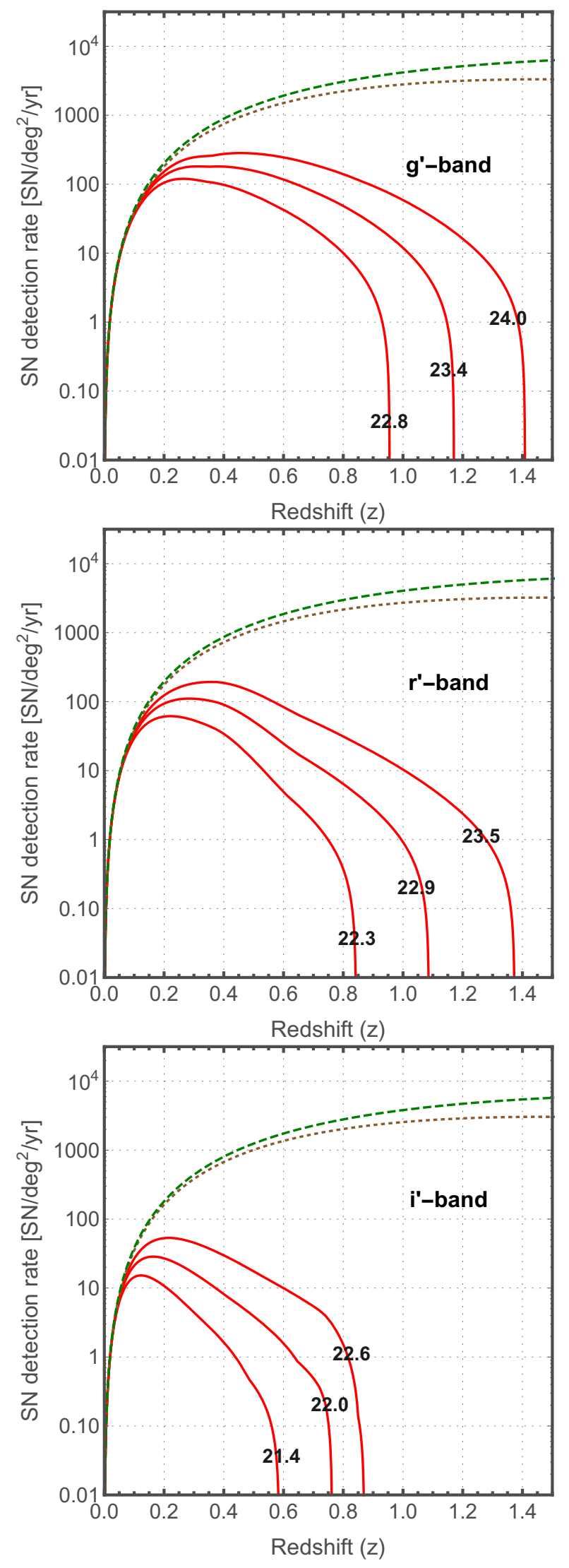

Figure 4. Detection rate of CCSN as a function of redshift. The dashed (green colour) and dotted (brown colour) curves, respectively indicate the cosmic CCSN rate without and with dust extinction consideration. Possible number of CCSNe to be detected with the ILMT in different bands $\left(g^{\prime}, r^{\prime}\right.$ and $i^{\prime}$ ) and for different magnitude limits (c.f. stacking of consecutive night images, see Section 3) are also shown. 

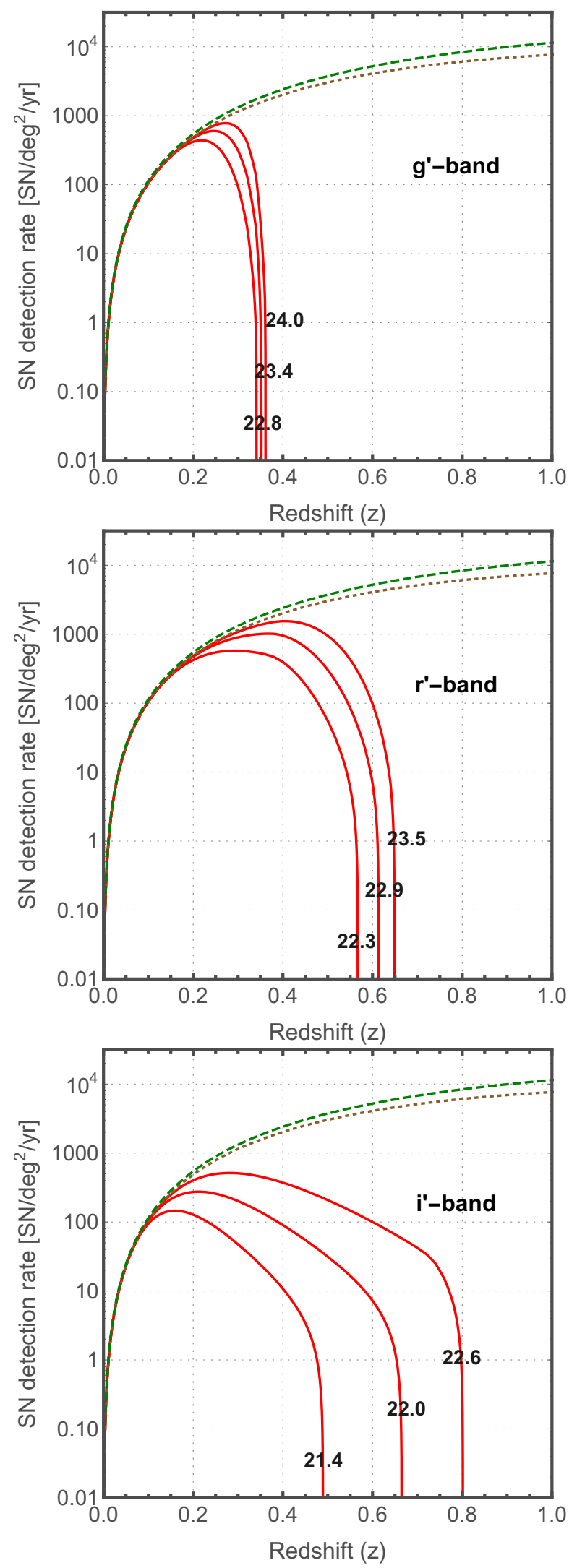

Figure 5. Detection rate of Type Ia SN as a function of redshift. The curves are similar to those in Fig. 4 but for Type Ia SNe.

which can be written as

$$
R_{\mathrm{SN}}(z)=\frac{X_{\mathrm{SN}}}{\langle m\rangle_{\mathrm{SN}}} \dot{\rho}_{\star}(z)
$$

where $X_{S N}$ is the fraction of stellar mass which results in supernovae $\left(=\int_{S N} M \xi(M) d M / \int M \xi(M) d M\right),\langle m\rangle_{\mathrm{SN}}$ is the average supernova progenitor mass $\left(=\int_{S N} M \xi(M) d M / \int \xi(M) d M\right)$ and $\dot{\rho}_{\star}(z)$ is the star formation rate. $\xi(M)$ represents the stellar initial mass function (IMF). The quantity $f_{\text {detect }}(z)$ is the fraction of $\mathrm{SNe}$ which can be detected by the instrument and it depends on the characteristics of the instrument and the SN type (i),

$$
f_{\text {detect }}(z)=f_{\text {dust }}(z) \times \frac{1}{N} \sum_{i=1}^{N} \int_{-\infty}^{m_{\text {lim }, x}} \phi_{i}(m, z) d m
$$

$i$ denotes different SN types, for example in case of CCSNe we considered all four types, Ibc (both Ib and Ic), IIL, IIP and IIn (i.e. $\mathrm{N}=4)$, whereas for Type Ia SNe, $\mathrm{N}=1$. The $\phi_{i}(m, z)$ is the supernova luminosity function or the magnitude distribution function which is assumed to be a normal distribution with the mean magnitude $\tilde{m}_{i}$ and variance $\sigma_{i}$, where $\tilde{m}_{i}=\tilde{m}_{i}^{a b s}+5 \log \left[d_{L}(z) / 10 \mathrm{pc}\right]+$ $K_{i x}(z)+\eta_{i x B}+A(z) ; d_{L}(z)$ is the luminosity distance, $K_{i x}(z)$ is the $K$-correction, $\eta_{i x B}$ is the color correction and $A(z)$ is the dust correction (for details see, Lien \& Fields 2009).

The $K$-correction and the color correction can be computed as follows,

$$
\begin{aligned}
K_{i x}(z) & =2.5 \log (1+z)+2.5 \log \frac{\int F_{i}(\lambda) S_{x}(\lambda) d \lambda}{\int F_{i}(\lambda /(1+z)) S_{x}(\lambda) d \lambda} \\
\eta_{i x B} & =\quad-2.5 \log \frac{\int F_{i}(\lambda) S_{x}(\lambda) d \lambda}{\int F_{i}(\lambda) S_{B}(\lambda) d \lambda}+\text { zeropoint correction }
\end{aligned}
$$

here, $F(\lambda)$ is the intrinsic spectral distribution of the supernova, $S_{x}(\lambda)$ and $S_{B}(\lambda)$ are the response functions of the filter $x$ (ILMT filter bands) and $B$ (filter band used to estimate the absolute magnitude distribution), respectively (for more details see Lien \& Fields 2009).

We have considered a flat cosmology with $\Omega_{m}=0.31$, and $h=0.68$ which are consistent with the recent Planck results (Planck Collaboration XIII 2016). The absolute magnitude distribution of the SNe is taken from Richardson et al. (2014) and has been adjusted for the Hubble parameter value $h=0.68$. We also put a conservative cut-off at $2.5 \sigma$ in the absolute magnitude distribution as there are no data available beyond $2.5 \sigma$ in the sample of Richardson et al. (2014). We take the same IMF, $\dot{\rho}_{\star}(z)$ and the dust correction as given by Lien \& Fields (2009). The progenitor mass range for the Type Ia $\mathrm{SNe}$ is taken to be $3-8 \mathrm{M}_{\odot}$ and for $\mathrm{CC}$ $\mathrm{SNe}$ it is taken to be $8-50 \mathrm{M}_{\odot}$ (mass range for all four CCSNe types i.e. Ibc, IIL, IIP and IIn). Further, for CCSNe the form $F(\lambda)$ is taken as the one listed in Lien \& Fields (2009), and for Type Ia SNe we choose $F(\lambda)$ as a $15000 \mathrm{~K}$ blackbody spectrum with cutoff due to UV blanketing at $\lambda<4000 \AA$ (Dahlén \& Fransson 1999; Foley \& Kasen 2011). For the calculation of magnitude limited detection rate we have used the expected magnitude limits $\left(m_{\text {lim }, x}\right)$ for the ILMT in the different spectral bands (see Section 3).

In Figs. 4 and 5, the dotted-brown and the dashed-green lines show the cosmic supernova rate with and without dust correction, respectively. The solid-red lines correspond to the expected magnitude limited SN detection rate observed with the ILMT. The set of three magnitude limits respectively correspond to 1, 3 and 6 night integration time (see Section 3).

The cut-off in the absolute magnitude distribution has a very 
Table 2. SN detection rates with the ILMT. $1_{N}, 3_{N}$ and $6_{N}$ indicate the number of $\mathrm{SNe}$ for the limiting magnitudes of single, and co-added images of 3 and 6 nights, respectively. Total number of SNe (columns 6, 7 and 8) are the redshift integrated events in a year (only 160 photometric nights of the site and an average 8 hours of observing time each night have been accounted for).

\begin{tabular}{cccccccc}
\hline SN & Filter & \multicolumn{3}{c}{ SNe/deg 2 year } & \multicolumn{3}{c}{ Total SNe in a year } \\
Type & & $1_{\mathrm{N}}$ & $3_{\mathrm{N}}$ & $6_{\mathrm{N}}$ & $1_{\mathrm{N}}$ & $3_{\mathrm{N}}$ & $6_{\mathrm{N}}$ \\
\hline Ia & $g^{\prime}$ & 63 & 89 & 115 & 1299 & 1835 & 2371 \\
& $r^{\prime}$ & 155 & 274 & 426 & 3196 & 5649 & 8783 \\
& $i^{\prime}$ & 28 & 71 & 174 & 577 & 1464 & 3588 \\
& & & & & & & \\
$\mathrm{CC}$ & $g^{\prime}$ & 50 & 97 & 177 & 1031 & 2000 & 3649 \\
& $r^{\prime}$ & 20 & 43 & 87 & 412 & 887 & 1794 \\
& $i^{\prime}$ & 3 & 8 & 19 & 62 & 165 & 392 \\
\hline
\end{tabular}

strong effect on the high redshift cut-off seen in the magnitude limited detection rate plots. Changing the cut-off from $2.5 \sigma$ to $3.0 \sigma$ increases the cutoff redshift drastically, though it does not change the total supernovae counts by much and it has little effect over the redshift value corresponding to the maximum detection rate as has also been pointed out by Lien \& Fields (2009). Setting a cut-off in the absolute magnitude distribution at $2.5 \sigma$ gives a good, yet conservative, estimate for the detection rate.

The sharp cut-off seen in the case of Type Ia SNe (Fig. 5, $g^{\prime}$-band) is due to the UV blanketing effect that cuts-off the spectral energy distribution at $z \gtrsim \lambda / 4000$. It makes the $\mathrm{K}$-correction very high for $z \gtrsim \lambda / 4000$ (see Fig. A1; $g^{\prime}$-band SN-Ia), making $\tilde{m}_{\mathrm{Ia}}(z \gtrsim \lambda / 4000) \gg m_{\text {lim, } g^{\prime}}$. As a result we get a sharp cut-off at a comparatively lower redshift in the $g^{\prime}$-band for Type Ia SNe.

Ground based observing facilities are sometimes affected by local weather and the ILMT site is not an exception. The wet and humid conditions during the Monsoon season necessitate the closure of all observing facilities situated at the site from June to September every year. Therefore, ILMT observations will also be closed during that period (i.e. 4 months). Previous observing experiences suggest that in general, the Devasthal site has $\sim 210$ clear nights in a year, among that $\sim 160$ nights are of photometric quality (see Sagar et al. 2000). Taking into account the site photometric nights and the area covered by the ILMT each night, we have estimated total number of possible $\mathrm{SNe}$ to be detected with this facility. These numbers are listed in Table 2 (see also Table B1). It is obvious that the above-estimated $\mathrm{SNe}$ numbers will reasonably vary during real observations if we also consider the night limitations and technical difficulties/maintenance of the instruments. The uncertainties in the above estimates are therefore not discussed further.

\subsection{Detection of supernova candidates}

The ILMT pointing is fixed towards the best seeing and atmospheric transparency position (i.e. at zenith). This allows one to obtain images with optimal quality. During each clear night, the same strip of sky will be scanned by the telescope. To detect SNe, previous night images or a good reference image will be subtracted from the search night images. We plan to perform automated realtime data reduction pipeline based on the Optimal Image Subtraction (OIS) technique (Alard \& Lupton 1998; Alard 2000).

In order to detect $\mathrm{SNe}$ and classify their type, utmost care is needed. Miscellaneous astrophysical and/or non-astrophysical con- taminating sources such as variable stars, quasars, active galaxies, asteroids, cosmic rays, etc. may appear on the acquired images. These unwanted sources must be removed accurately to avoid false detection. Various catalogues for different types of variable sources can be used to cross-match newly discovered transient candidates. Some catalogues are: VERONCAT ${ }^{5}$ (Véron-Cetty \& Véron 2010) for quasars and AGN, Minor Planet Checker ${ }^{6}$ for planets \& comets, and SIMBAD $^{7}$ (Wenger et al. 2000) for variable stars. Following the detection of a possible SN candidate, information will be communicated to the astronomical community (e.g. through ATEL and CBET), and also it will be available on the ILMT webpage.

The appearance of emission lines in the spectra is mandatory for classification and confirmation of a supernova. The spectrum is also useful to estimate the redshift and age (time after explosion) of SNe. However, distant $\mathrm{SNe}$ may be too faint for spectroscopy. Moreover, the follow-up spectroscopy will be a virtually impossible approach considering the large amount of transients detected in surveys. Therefore, new techniques have been developed attributing to $\mathrm{SNe}$ photometric classification. These are primarily based on some form of light curve fitting models e.g. MLCS/MLCS2k2 ${ }^{8}$ (Riess et al. 1995; Jha et al. 2007) and SALT/SALT2 ${ }^{9}$ (Guy et al. 2005, 2007). The observed data points are fitted to the templates and the most likelihood type is determined (see, e.g. Poznanski et al. 2002, 2007; Gal-Yam et al. 2004; Sullivan et al. 2006; Johnson \& Crotts 2006; Kuznetsova \& Connolly 2007; Kunz et al. 2007; Rodney \& Tonry 2009; Gong et al. 2010; Falck et al. 2010; Sako et al. 2011). The above cited models along with colour cuts and colour evolution based techniques (e.g. Dahlen \& Goobar 2002; Johnson \& Crotts 2006) can be applied for the type determination of the ILMT discovered SNe.

A quick and dense monitoring (particularly, during the phase just after the explosion to a few weeks after the explosion) of $\mathrm{SNe}$ will help in the type identification more accurately. Furthermore, at the moment of shock break-out, some SNe are expected to emit a short burst of high energy radiation (Nakar \& Sari 2010). Thereafter, the shock break-out cooling may create an early peak in the optical passband (Piro \& Nakar 2013). The early phase observations are therefore crucial to constrain the progenitor system (Bersten et al. 2012; Taddia et al. 2015). Here we emphasize that the ILMT will work in the continuous data acquisition mode. Accordingly, while it will contribute with data on each night basis nonetheless, it will not be possible to observe again a particular sky patch on the same night once it has passed over the ILMT field of view. Additionally, due to the limitation of the ILMT filter system it will be difficult to obtain precise colour and light curve information of SN candidates. Therefore, complementary observations with conventional glass mirror telescopes will be very useful. Thanks to the ARIES observing facilities which host three modern glass telescopes with different apertures (the 1.04-m Sampurnanad Telescope (ST), the 1.3-m DFOT and 3.6-m DOT). Depending upon the brightness and peculiarity of the newly discovered objects these telescopes along with other existing observing facilities in India and worldwide can be triggered for follow-up observations. A detailed follow-up scheme is presented in Kumar et al. (2018).

\footnotetext{
${ }^{5}$ https://heasarc.gsfc.nasa.gov/w3browse/all/veroncat. html

${ }^{6}$ http://www.minorplanetcenter.net

7 http://simbad.u-strasbg.fr/simbad/

${ }^{8}$ Multicolour light-curve shape

9 Spectral adaptive light curve template
} 
It is important to mention that along with the SN studies, the ILMT has other scientific interests also. That includes surveys for multiply imaged quasars, determination of trigonometric parallaxes of faint nearby objects, detection of high stellar proper motions and short to long term photometric variability studies of stellar objects (Surdej et al. 2006, 2018). Therefore, a reasonable balance between different filters is a must. The $i^{\prime}$ filter can be placed around the bright moon phases and during the remaining nights a combination of $g^{\prime}, r^{\prime}$ and $i^{\prime}$ filters can be placed alternatively. Such an observing strategy may equally be useful for $\mathrm{SN}$ candidate detection and other science cases as well.

\section{SUMMARY AND CONCLUSIONS}

The redshift-integrated supernova rate may turn out to be very large ( $\simeq 5-15$ events/sec, Madau et al. 1998). Considering their random occurrence in the Universe, it is not feasible to detect and observe each event. Monitoring all of them is also almost impossible as it will require a significant amount of telescope time. On the other hand, a regular imaging of a same strip of sky with the ILMT will be advantageous to apply the image subtraction technique for detecting transients such as SNe. Moreover, once a SN is detected in the ILMT images, it will by default provide dense sampled (successive night) light curves in different filters. The single scan ILMT limiting magnitudes are $\sim 22.8, \sim 22.3$ and $\sim 21.4$ mag in $g^{\prime}, r^{\prime}$ and $i^{\prime}$ filters, respectively and can be increased if we co-add the images taken on different nights. In this way, the ILMT survey should play an important role in $\mathrm{SNe}$ detection up to reasonably fainter limits with a precise and unbiased imaging of a strip of sky at a declination equal to the latitude of Devasthal. We are expecting to detect hundreds of Type Ia as well as core-collapse SNe up to intermediate redshifts thanks to the ILMT survey (c.f. Table 2). The multi-band and well sampled observations should enable the photometric type determination (by template fitting, colour information) of SNe more accurately. The expected large SNe samples yielded from the ILMT may also increase the representative objects of each type with better statistics. Furthermore, the ILMT will provide an untargeted search with plentiful of anonymous galaxies in each night images, which may allow us to construct a SN sample without host-galaxy biases in a given limited patch of sky.

The observational properties and theoretical modelling indicate that supernova light curves are mainly powered by a combination of two energy sources, i.e. shock generated energy deposition and radioactive decay ${ }^{56} \mathrm{Ni} \rightarrow{ }^{56} \mathrm{Co} \rightarrow{ }^{56} \mathrm{Fe}$, synthesized in the explosion (see Hoyle \& Fowler 1960; Colgate \& McKee 1969; Arnett 1982). In some of the $\mathrm{SNe}$, circumstellar interactions (Renzini 1978; Chugai 1991; Chevalier \& Fransson 1994) and rapidly rotating magnetars (Kasen \& Bildsten 2010; Woosley 2010; Dessart et al. 2012) can also supply energy. The light curves of different types of $\mathrm{SNe}$ exhibited diverse characteristics, e.g. Type II SNe: (Arcavi et al. 2012; Anderson et al. 2014; Sanders et al. 2015), stripped envelope $\mathrm{SNe}^{10}$ : (Drout et al. 2011; Bianco et al. 2014; Taddia et al. 2015; Lyman et al. 2016; Prentice et al. 2016) and Type Ia SNe: (Hayden et al. 2010; Ashall et al. 2016; Hoeflich et al. 2017, and references therein). The high quality light curves can provide an opportunity to robustly determine various parameters and empirical relations including the

10 In these events, the outer envelopes of hydrogen and/or helium of their progenitors are partially or completely removed before the explosion (e.g. Type IIb, Ib, Ic, and Ic-BL). 'rise time', the light curve decline rate parameter $\left(\Delta \mathrm{m}_{15}\right.$, Phillips 1993), and the color evolution etc. of different types of SNe. This will also allow us to use theoretical models for a robust determination of the explosion parameters (e.g. the ${ }^{56} \mathrm{Ni}$ mass synthesized, the ejected mass and explosion energy). That will in turn shed some light on the explosion mechanisms of different $\mathrm{SNe}$ and evolutionary stages of their progenitors.

It is noteworthy that the spectra of SNe provide crucial information about the composition and distribution of elements in the ejecta. Therefore, the spectroscopic monitoring of peculiar events will also be very valuable. In this context, a guaranteed-time allocation strategy on 3.6-m DOT to follow-up newly discovered objects will fulfill our needs. Because of the tight link between $\mathrm{SNe}$ and star formation, the ILMT with complementary observations and along with other sky surveys (e.g. Large Synoptic Survey Telescope (LSST), ZTF, etc.) may provide better measurements of the moderate redshift history of the cosmic star-formation rate. New SNe discoveries and precise investigation of their light curves could improve our knowledge on a variety of problems including cosmology and SN physics.

\section{ACKNOWLEDGMENTS}

The authors thank the referee for his/her useful comments that substantially improved this paper. We are grateful to the members of the ILMT team who provided their sincere efforts for this project that is now in its final stage of installation. Although the list of ILMT contributors is long, we specially thank J. P. Swings, Ram Sagar, Hum Chand, Ludovic Delchambre, Serge Habraken and Bikram Pradhan for their precious support in this project. Active involvement of Anna Pospieszalska is highly acknowledged. BK and KLP also thank Amy Lien for fruitful discussions during the preparation of this manuscript. BK acknowledges the Science and Engineering Research Board (SERB) under the Department of Science \& Technology, Govt. of India, for financial assistance in the form of National Post-Doctoral Fellowship (Ref. no. PDF/2016/001563). SBP acknowledges BRICS grant DST/IMRCD/BRICS/Pilotcall/ProFCheap/2017(G) for the present work. This research has also been supported by Région Wallonne (Belgium) under the convention 516238, F.R.S.-FNRS (Belgium), the Liège University and the Natural Sciences and Engineering Research Council of Canada. JS wishes to express his special thanks to Ir. Alain Gillin, Director, for his comprehension in renewing the Convention no 516238 'TML4M' during many years and Prof. Govind Swarup, former Chair of the ARIES Governing Council, for his constant and very important support. Part of this work was initiated during the doctoral thesis of Brajesh Kumar.

\section{REFERENCES}

Abbott T., et al., 2005, preprint, (arXiv:0510346)

Alard C., 2000, A\&AS, 144, 363

Alard C., Lupton R. H., 1998, ApJ, 503, 325

Anderson J. P., et al., 2014, ApJ, 786, 67

Arcavi I., et al., 2012, ApJ, 756, L30

Arnett W. D., 1982, ApJ, 253, 785

Ashall C., Mazzali P., Sasdelli M., Prentice S. J., 2016, MNRAS, 460, 3529

Astier P., et al., 2006, A\&A, 447, 31

Bellm E., 2014, in Wozniak P. R., Graham M. J., Mahabal A. A., Seaman R., eds, The Third Hot-wiring the Transient Universe Workshop. pp 27-33 (arXiv: 1410.8185$)$ 
Bersten M. C., et al., 2012, ApJ, 757, 31

Bianchi S., Schneider R., 2007, MNRAS, 378, 973

Bianco F. B., et al., 2014, ApJS, 213, 19

Borra E. F., 1982, JRASC, 76, 245

Borra E. F., 2001b, preprint, (arXiv: 0102432)

Borra E. F., 2001a, preprint, (arXiv:0102064)

Borra E. F., 2003, A\&A, 404, 47

Borra E. F., Beauchemin M., Arsenault R., Lalande R., 1985, PASP, 97, 454

Borra E. F., Content R., Drinkwater M. J., Szapiel S., 1989, ApJ, 346, L41

Borra E. F., Content R., Delisle C., Gauvin J., 1992a, PASP, 104, 1239

Borra E. F., Content R., Girard L., Szapiel S., Tremblay L. M., Boily E., 1992b, ApJ, 393, 829

Botticella M. T., et al., 2017, A\&A, 598, A50

Branch D., Tammann G. A., 1992, ARA\&A, 30, 359

Branch D., Nomoto K., Filippenko A. V., 1991, Comments on Astrophysics, 15,221

Cappellaro E., et al., 2015, A\&A, 584, A62

Chandrasekhar S., 1931, ApJ, 74, 81

Chevalier R. A., Fransson C., 1994, ApJ, 420, 268

Chugai N. N., 1991, MNRAS, 250, 513

Colgate S. A., McKee C., 1969, ApJ, 157, 623

Cucciati O., et al., 2012, A\&A, 539, A31

Dahlén T., Fransson C., 1999, A\&A, 350, 349

Dahlen T., Goobar A., 2002, PASP, 114, 284

Dahlen T., et al., 2004, ApJ, 613, 189

Deep A., Fiorentino G., Tolstoy E., Diolaiti E., Bellazzini M., Ciliegi P., Davies R. I., Conan J.-M., 2011, A\&A, 531, A151

Dessart L., Hillier D. J., Waldman R., Livne E., Blondin S., 2012, MNRAS, 426, L76

Dilday B., et al., 2008, ApJ, 682, 262

Drake A. J., et al., 2009, ApJ, 696, 870

Drout M. R., et al., 2011, ApJ, 741, 97

Elmegreen B. G., 1998, in Woodward C. E., Shull J. M., Thronson Jr. H. A., eds, Astronomical Society of the Pacific Conference Series Vol. 148, Origins. p. 150

Falck B. L., Riess A. G., Hlozek R., 2010, ApJ, 723, 398

Filippenko A. V., 1997, ARA\&A, 35, 309

Filippenko A. V., 2005, in Sion E. M., Vennes S., Shipman H. L., eds, Astrophysics and Space Science Library Vol. 332, White dwarfs: cosmological and galactic probes. pp 97-133

Filippenko A. V., Li W. D., Treffers R. R., Modjaz M., 2001, in Paczynski B., Chen W.-P., Lemme C., eds, ASP Conf. Ser.Vol. 246, IAU Colloq. 183: Small Telescope Astronomy on Global Scales. p. 121

Finet F., 2013, in PhD Thesis. University of Liège, Belgium. pp 13-170

Foley R. J., Kasen D., 2011, ApJ, 729, 55

Frieman J. A., et al., 2008, AJ, 135, 338

Gal-Yam A., Poznanski D., Maoz D., Filippenko A. V., Foley R. J., 2004, PASP, 116, 597

Gibson B. K., Hickson P., 1992, MNRAS, 258, 543

Gong Y., Cooray A., Chen X., 2010, ApJ, 709, 1420

Graur O., et al., 2011, MNRAS, 417, 916

Guy J., Astier P., Nobili S., Regnault N., Pain R., 2005, A\&A, 443, 781

Guy J., et al., 2007, A\&A, 466, 11

Hamuy M., et al., 2006, PASP, 118, 2

Hayden B. T., et al., 2010, ApJ, 712, 350

Heger A., Fryer C. L., Woosley S. E., Langer N., Hartmann D. H., 2003, ApJ, 591, 288

Herbst W., Assousa G. E., 1977, ApJ, 217, 473

Hickson P., Racine R., 2007, PASP, 119, 456

Hickson P., Richardson E. H., 1998, PASP, 110, 1081

Hickson P., Gibson B. K., Hogg D. W., 1993, PASP, 105, 501

Hickson P., Borra E. F., Cabanac R., Content R., Gibson B. K., Walker G. A. H., 1994, ApJ, 436, L201

Hickson P., et al., 2007, PASP, 119, 444

Hillebrandt W., Niemeyer J. C., 2000, ARA\&A, 38, 191

Hoeflich P., et al., 2017, ApJ, 846, 58

Hopkins A. M., 2004, ApJ, 615, 209

Hopkins A. M., Beacom J. F., 2006, ApJ, 651, 142
Howell S. B., 1989, PASP, 101, 616

Howell S. B., 2000, Handbook of CCD Astronomy

Hoyle F., Fowler W. A., 1960, ApJ, 132, 565

Humason M. L., Mayall N. U., Sandage A. R., 1956, AJ, 61, 97

Jha S., Riess A. G., Kirshner R. P., 2007, ApJ, 659, 122

Johnson B. D., Crotts A. P. S., 2006, AJ, 132, 756

Kaiser N., et al., 2002, in Tyson J. A., Wolff S., eds, Proc. SPIEVol. 4836, Survey and Other Telescope Technologies and Discoveries. pp 154-164 Kasen D., Bildsten L., 2010, ApJ, 717, 245

Kumar B., 2014, in PhD Thesis. University of Liège, Belgium. pp 3-289 (http://hdl.handle.net/2268/174851)

Kumar B., Surdej J., Hickson P., Borra E. F., Finet F., Swings J. P., Habraken S., Pandey S. B., 2015, in Astronomical Society of India Conference Series.

Kumar B., Pandey S. B., Pandey K. L., Anupama G. C., Surdej J., 2018, in Bulletin of Liège Royal Society of Sciences. University of Liège, Belgium

Kunz M., Bassett B. A., Hlozek R. A., 2007, Phys. Rev. D, 75, 103508

Kuznetsova N. V., Connolly B. M., 2007, ApJ, 659, 530

Langer N., 2012, ARA\&A, 50, 107

Law N. M., et al., 2009, PASP, 121, 1395

Le Floc'h E., et al., 2005, ApJ, 632, 169

Li W. D., et al., 2000, in Holt S. S., Zhang W. W., eds, AIP Conf. SerVol. 522, AIP Conf. Ser.. pp 103-106

Lien A., Fields B. D., 2009, J. Cosmology Astropart. Phys., 1, 47

Lyman J. D., Bersier D., James P. A., Mazzali P. A., Eldridge J. J., Fraser M., Pian E., 2016, MNRAS, 457, 328

Madau P., della Valle M., Panagia N., 1998, MNRAS, 297, L17

Magette A., 2010, in PhD Thesis. University of Liège Belgium. pp 7-253

Maoz D., Mannucci F., Nelemans G., 2014, ARA\&A, 52, 107

Matteucci F., Greggio L., 1986, A\&A, 154, 279

Mayya Y. D., 1991, JoAA, 12, 319

McLean I. S., 1989, Electronic and computer-aided astronomy: From eyes to electronic sensors

Miknaitis G., et al., 2007, ApJ, 666, 674

Minkowski R., 1941, PASP, 53, 224

Nakar E., Sari R., 2010, ApJ, 725, 904

Neill J. D., et al., 2006, AJ, 132, 1126

Oke J. B., Sandage A., 1968, ApJ, 154, 21

Pain R., et al., 1996, ApJ, 473, 356

Perlmutter S., et al., 1999, ApJ, 517, 565

Perrett K., et al., 2012, AJ, 144, 59

Phillips M. M., 1993, ApJ, 413, L105

Piro A. L., Nakar E., 2013, ApJ, 769, 67

Planck Collaboration et al., 2016, A\&A, 594, A13

Podsiadlowski P., Joss P. C., Hsu J. J. L., 1992, ApJ, 391, 246

Poels J., et al., 2012, in Griffin E., Hanisch R., Seaman R., eds, IAU Symposium Vol. 285, IAU Symposium. pp 394-396

Poznanski D., Gal-Yam A., Maoz D., Filippenko A. V., Leonard D. C., Matheson T., 2002, PASP, 114, 833

Poznanski D., Maoz D., Gal-Yam A., 2007, AJ, 134, 1285

Pradhan B., et al. 2018, in Bulletin of Liège Royal Society of Sciences. University of Liège, Belgium

Prentice S. J., et al., 2016, MNRAS, 458, 2973

Pritchet C. J., Howell D. A., Sullivan M., 2008, ApJ, 683, L25

Puls J., Vink J. S., Najarro F., 2008, A\&A Rev., 16, 209

Renzini A., 1978, Mem. Soc. Astron. Italiana, 49, 389

Richardson D., Branch D., Casebeer D., Millard J., Thomas R. C., Baron E., 2002, AJ, 123, 745

Richardson D., Branch D., Baron E., 2006, AJ, 131, 2233

Richardson D., Jenkins III R. L., Wright J., Maddox L., 2014, AJ, 147, 118

Riess A. G., Press W. H., Kirshner R. P., 1995, ApJ, 438, L17

Riess A. G., et al., 1998, AJ, 116, 1009

Riess A. G., et al., 2007, ApJ, 659, 98

Rodney S. A., Tonry J. L., 2009, ApJ, 707, 1064

Rujopakarn W., et al., 2010, ApJ, 718, 1171

Sagar R., 2016, preprint, (arXiv: 1607.06455)

Sagar R., et al., 2000, A\&AS, 144, 349 
Sagar R., et al., 2011, Current Science, 101, 8

Sagar R., Kumar B., Omar A., Joshi Y. C., 2012, in Astron. Soc. of India Conf. Ser. p. 173

Sako M., et al., 2008, AJ, 135, 348

Sako M., et al., 2011, ApJ, 738, 162

Sana H., et al., 2012, Science, 337, 444

Sanders N. E., et al., 2015, ApJ, 799, 208

Schiminovich D., et al., 2005, ApJ, 619, L47

Shappee B. J., et al., 2014, ApJ, 788, 48

Smartt S. J., 2009, ARA\&A, 47, 63

Smith N., 2014, ARA\&A, 52, 487

Smith N., Owocki S. P., 2006, ApJ, 645, L45

Sullivan M., et al., 2006, AJ, 131, 960

Surdej J., et al., 2006, in SPIE Conf. Ser. Vol. 6267.

Surdej J., et al. 2018, in Bulletin of Liège Royal Society of Sciences. University of Liège, Belgium

Taddia F., et al., 2015, A\&A, 574, A60

Taylor M., et al., 2014, ApJ, 792, 135

Todini P., Ferrara A., 2001, MNRAS, 325, 726

Vangeyte B., Manfroid J., Surdej J., 2002, A\&A, 388, 712

Véron-Cetty M.-P., Véron P., 2010, A\&A, 518, A10

Wang B., Han Z., 2012, New A Rev., 56, 122

Wenger M., et al., 2000, A\&AS, 143, 9

Wheeler J. C., Levreault R., 1985, ApJ, 294, L17

Whelan J., Iben Jr. I., 1973, ApJ, 186, 1007

Wood-Vasey W. M., et al., 2007, ApJ, 666, 694

Woosley S. E., 2010, ApJ, 719, L204

Woosley S. E., Taam R. E., Weaver T. A., 1986, ApJ, 301, 601

Yoon S.-C., Woosley S. E., Langer N., 2010, ApJ, 725, 940

\section{APPENDIX A: $K$-CORRECTION}

The K-correction term accounts for the conversion from an observed magnitude to that which would be observed in the rest frame in another passband (Humason et al. 1956; Oke \& Sandage 1968). Fig. A1 represents K-corrections for different $\mathrm{SNe}$ that have been applied to calculate ILMT SNe rate for the $g^{\prime}, r^{\prime}$ and $i^{\prime}$ filters.

\section{APPENDIX B: PREDICTED SN WITH THE ILMT}

In Table B1, the predicted SN rate (for Ia and CC) estimated for $\Delta z$ $=0.2$ bins are listed.
Table B1. Predicted Type Ia and CCSNe (/deg $2 /$ year) in $\Delta z=0.2$ bins. $1_{\mathrm{N}}$, $3_{\mathrm{N}}$ and $6_{\mathrm{N}}$ indicate the number of $\mathrm{SNe}$ for the limiting magnitudes of single, and co-added images of 3 and 6 nights, respectively.

\begin{tabular}{|c|c|c|c|c|c|c|c|}
\hline \multirow{2}{*}{$\begin{array}{l}\text { Redshift } \\
(z)\end{array}$} & \multirow[t]{2}{*}{ Filter } & \multicolumn{3}{|c|}{ Ia SNe } & \multicolumn{3}{|c|}{ CCSNe } \\
\hline & & $1_{\mathrm{N}}$ & $3_{\mathrm{N}}$ & $6_{N}$ & $1_{\mathrm{N}}$ & $3_{N}$ & $6_{N}$ \\
\hline 0.2 & & 29 & 30 & 30 & 8 & 10 & 10 \\
\hline 0.4 & & 63 & 89 & 115 & 31 & 44 & 57 \\
\hline 0.6 & & 63 & 89 & 115 & 45 & 75 & 111 \\
\hline 0.8 & $g^{\prime}$ & 63 & 89 & 115 & 50 & 91 & 150 \\
\hline 1.0 & & 63 & 89 & 115 & 50 & 96 & 169 \\
\hline 1.2 & & 63 & 89 & 115 & 50 & 97 & 176 \\
\hline 1.4 & & 63 & 89 & 115 & 50 & 97 & 177 \\
\hline 0.2 & & 29 & 30 & 30 & 06 & 08 & 09 \\
\hline 0.4 & & 133 & 199 & 243 & 16 & 29 & 44 \\
\hline 0.6 & & 155 & 274 & 424 & 20 & 39 & 71 \\
\hline 0.8 & $r^{\prime}$ & 155 & 274 & 426 & 20 & 42 & 82 \\
\hline 1.0 & & 155 & 274 & 426 & 20 & 43 & 86 \\
\hline 1.2 & & 155 & 274 & 426 & 20 & 43 & 87 \\
\hline 1.4 & & 155 & 274 & 426 & 20 & 43 & 87 \\
\hline 0.2 & & 17 & 24 & 28 & 02 & 04 & 05 \\
\hline 0.4 & & 28 & 63 & 122 & 03 & 07 & 14 \\
\hline 0.6 & & 28 & 71 & 165 & 03 & 08 & 18 \\
\hline 0.8 & $i^{\prime}$ & 28 & 71 & 174 & 03 & 08 & 19 \\
\hline 1.0 & & 28 & 71 & 174 & 03 & 08 & 19 \\
\hline 1.2 & & 28 & 71 & 174 & 03 & 08 & 19 \\
\hline 1.4 & & 28 & 71 & 174 & 03 & 08 & 19 \\
\hline
\end{tabular}



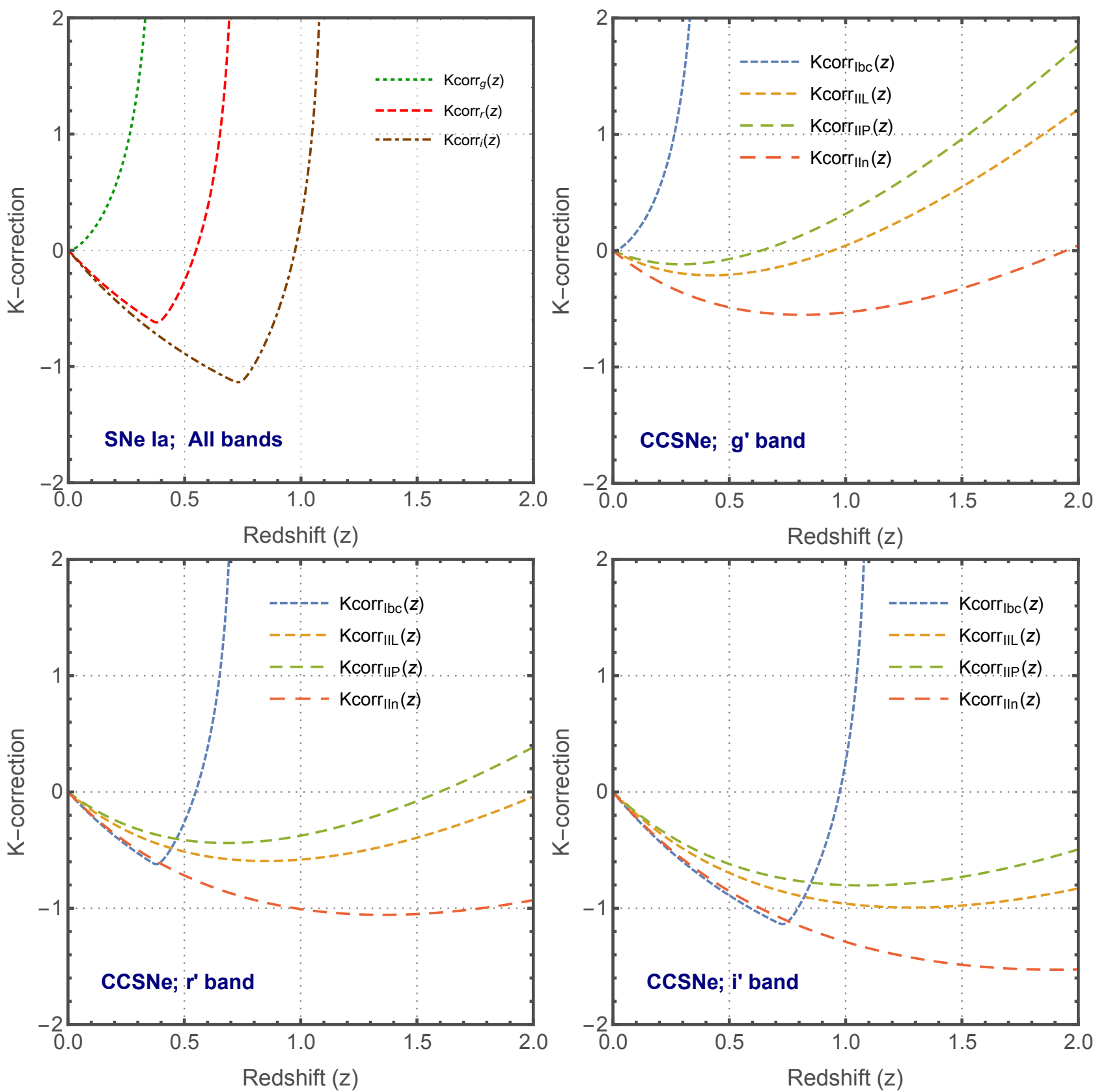

Figure A1. $K$-correction plots for the different Types of SNe (Ia, Ibc, IIL, IIP and IIn) in $g^{\prime}, r^{\prime}$ and $i^{\prime}$ bands. 